\title{
Non-invasive imaging of actinic cheilitis and squamous cell carcinoma of the lip
}

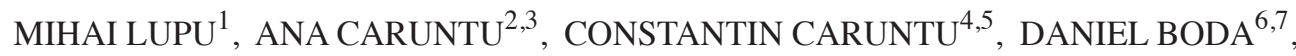 \\ LILIANA MORARU ${ }^{2}$, VLAD VOICULESCU $^{8}$ and ALEXANDRA BASTIAN ${ }^{9,10}$
}

\author{
${ }^{1}$ Department of Dermatology, MEDAS Medical Center, Bucharest 030442; ${ }^{2 ،}$ Carol Davila' Central Military \\ Emergency Hospital, Bucharest 010825; ${ }^{3}$ 'Titu Maiorescu’ University, Faculty of Medicine, Bucharest 031593; \\ ${ }^{4}$ Department of Physiology, 'Carol Davila' University of Medicine and Pharmacy, Bucharest 050474; ${ }^{5}$ Department of \\ Dermatology, 'Prof. N. Paulescu' National Institute of Diabetes, Nutrition and Metabolic Diseases, Bucharest 011233; \\ ${ }^{6}$ Dermatology Research Laboratory, 'Carol Davila' University of Medicine and Pharmacy, Bucharest 050474; ${ }^{7}$ Carol \\ Medical Center, Bucharest 010626; ${ }^{8}$ Department of Dermatology and Allergology, Elias Emergency University \\ Hospital, Bucharest 011461; ${ }^{9}$ Department of Pathology, 'Carol Davila' University of Medicine and Pharmacy, \\ Bucharest 050474; ${ }^{10}$ Department of Pathology, 'Colentina’ University Hospital, Bucharest 020125, Romania
}

Received December 19, 2017; Accepted February 22, 2018

DOI: $10.3892 / \mathrm{mco} .2018 .1599$

\begin{abstract}
An early diagnosis is of overwhelming importance for the management and prognosis of mucocutaneous cancer. Actinic cheilitis (AC), defined by the clonal expansion of genomically unstable keratinocytes, is the most common potentially malignant lesion affecting the lips. Squamous cell carcinoma (SCC) is the most frequent oral malignancy, and there is strong evidence that the majority of the SCCs of the lip originate from AC. There is considerable difficulty in discerning between dysplasia and invasive carcinomas solely on a clinical basis. Although dermoscopy has become an essential tool for skin tumor evaluation, reflectance confocal microscopy (RCM) is a non-invasive imaging technology that has proved itself extremely useful in the diagnosis and monitoring of several skin diseases, including AC and SCC. The present study aimed to re-emphasize the usefulness of RCM in the early detection of malignant transformation, using $\mathrm{AC}$ and SCC of the lips as working examples. Due to the apparent innocuousness of $\mathrm{AC}$ for numerous patients, it is not possible to overstress the importance of a correct and early diagnosis, proper treatment and long-term patient follow-up as being essential for preventing the progression to lip SCC, or for its timely diagnosis.
\end{abstract}

Correspondence to: Dr Caruntu Constantin, Department of Physiology, 'Carol Davila' University of Medicine and Pharmacy, 8 Eroii Sanitari Avenue, 050474 Bucharest, Romania

E-mail: costin.caruntu@gmail.com

Key words: actinic cheilitis, squamous cell carcinoma, microscopy, confocal, dermoscopy, early detection of cancer, lip neoplasms

\section{Introduction}

Actinic cheilitis (AC), a potentially malignant lesion (1), is the most frequently occurring, and among the most important, of the pathological processes that affect the lips. At the same time, oral and lip malignancy is the eighth most prevalent neoplastic disorder worldwide (2), with men being at far greater risk compared with women $(1.3 \%$ men vs. $0.3 \%$ women) (3-6). Although risk factors for carcinoma development are numerous [these include ultraviolet (UV) radiation exposure, smoking, premalignant lesions, several viruses, immunosuppression, and chronic trauma] (6,7), AC has been directly associated with chronic exposure to UV radiation, notably UVB radiation, due to its greater penetration potential when compared with UVA $(8,9)$. Albeit that occupational UV radiation exposure is a major risk factor for $\mathrm{AC}$ in men, its association with smoking and unhealthy eating habits may produce synergistic effects (10). Clinically, AC encompasses a broad spectrum of presentation, including pale, flaking or scaly lips, chronic ulcerations and erosions, white plaques, blurring of the demarcation between the lip vermillion border and the skin and vermillion atrophy, areas of erythema, and potentially other lesions (11-18). Palpation of the lesional surface produces a fine, 'sandpaper-like' feeling (18). While $\mathrm{AC}$ is usually asymptomatic, occasionally the affected person complains of lip dryness, a stinging or burning sensation, persistent scaling and impaired lip mobility (16), described as an inelastic or tight sensation in the lip (18). AC differential diagnosis includes several inflammatory lip disorders, such as lip eczema, cheilitis granulomatosa, benign leukoplakia, lichen planus, or straightforward dry skin and chronic irritation (19).

Squamous cell carcinoma (SCC) constitutes $\sim 90 \%$ of all cases of oral malignancy (20), and it is estimated that $95 \%$ of SCCs of the lip originate from AC $(21,22)$. Although the reported risk of actinic keratoses (AKs) progressing to SCC varies from $<1$ to $20 \%$ (16), the malignant transformation rate 
of AC into lip SCC is higher, and ranges from 10 to $30 \%$ (23). Clinically, the keratotic patches of AC may progress to thickening and induration, nodularity, and rapid growth, and eventually one or more of them ulcerate, causing bleeding and pain. Such changes are suggestive of AC progression to SCC of the lip $(9,10,18,24)$. Although cutaneous SCC originating from AKs has a relatively low rate of metastasis ( 0.53\%) (25), SCC of the lip is much more prone to metastasis (3-20\%) (26), and the risk of cervical lymph node metastatic spread is higher in lip SCC compared with cutaneous SCC, with lymph node disease detected 1-3 years after initial diagnosis and treatment in the case of SCC of the lips (10).

The difficulty of discriminating between mere dysplasias or invasive carcinomas is a constant therapeutic struggle, additionally complicated by the tendency towards field cancerization, inducing multicentric lesion sites (27). Several diagnostic techniques hold future promise for early detection, risk assessment and monitoring of skin cancer. Molecular analysis of the differences between the normal, inflammatory and malignant keratinocyte proteomes are likely to discover new biomarkers for SCC diagnosis, follow-up, and development of individualized targeted therapies for SCC and other cutaneous malignancies (28-30). Furthermore, more relevant for everyday clinical practice, dermoscopy and in vivo reflectance confocal microscopy (RCM) help further define the diagnostic and prognostic criteria of AC and SCC. RCM, a noninvasive imaging technology proving useful in the diagnosis of several skin diseases, has helped to bridge the gap between dermoscopy and histology (31-38). As with dermoscopy, RCM allows for in vivo horizontal enface examination of lesions, generating images of the epidermis and superficial dermis at resolutions close to those of optical light microscopy $(32,33)$. In epithelium, a resolution of $1 \mu \mathrm{m}$ with a field of view of $200-400 \mu \mathrm{m}$ and a penetration depth of $\sim 500 \mu \mathrm{m}$ have been achieved (31).

Early lesion detection and prompt, effective therapy still remain the most important determining factors of long-term patient survival and quality of life (39). Among the main reasons for using this non-invasive imaging technique is its ability to detect premalignant disorders such as AC, as well as skin and mucosal cancers at their earliest stage.

\section{Patients and methods}

After having given written informed consent, the two patients included in the present case report were subjected to the evaluation and treatment protocol described below.

Clinical evaluation. Patient examination for the presence of AC and/or SCC was based on clinical evaluations following guidelines for the visual inspection and diagnosis of skin cancer. Clinical photographs of skin and mucosal lesional sites were taken using a digital camera (Nikon D3300; Nikon Corporation, Tokyo, Japan).

Dermoscopy. In each case, dermoscopic images were acquired using a digital videodermoscopy system (FotoFinder, Bad Birnbach, Germany) and a VivaScope ${ }^{\circledR} 1500$ VivaCam macro-camera [Caliber Imaging \& Diagnostics, Inc. (formerly, Lucid Inc.), Rochester, NY, USA].
In vivo RCM imaging. A commercially available reflectance confocal microscope (VivaScope ${ }^{\circledR}$ 1500; Caliber Imaging \& Diagnostics, Inc.) was used for confocal imaging. A detailed description of this technique and the device used has been previously published $(35,40)$.

The two patients discussed in the present case report underwent classic vermilionectomy under local anesthesia in the Department of Oral and Maxillofacial Surgery of 'Dr Carol Davila' Central Military Hospital in Bucharest, with clear excision margins on histopathology. There were no post-operative complications, and sutures were removed in 10 days in both cases.

Histology. For the two patients featured in this case report, the excised tissues were subjected to histopathological examination using standard hematoxylin-eosin staining.

\section{Case reports}

Case 1. A 71-year-old Caucasian male was referred in 2015 from the Oral and Maxillofacial Surgery Department of 'Dr Carol Davila' Central Military Hospital in Bucharest to the Dermatology Department of 'Prof. N. C. Paulescu' National Institute of Diabetes, Nutrition and Metabolic Diseases, Bucharest, for the evaluation of multiple, asymptomatic milky-white keratotic areas on the surface of his lower lip. The patient reported significant occupational sun exposure throughout his lifetime, consistent with the presence of areas of mottled, telangiectatic, and lentiginous skin changes. A clinical examination revealed multiple white keratotic areas on an atrophic lower lip surface in conjunction with a blurred vermillion-skin contour (Fig. 1A). Dermoscopy revealed a milky-white plaque with well-defined borders, equivalent to hyperkeratosis, surrounded by telangiectatic and tortuous vessels (Fig. 1B). An RCM examination revealed areas of uneven tissular architecture with enlarged intercellular spaces and loss of the normal 'honeycomb' appearance (Fig. 1C and D). Dilated and tortuous vessels, and perivascular inflammatory cells with shiny appearance, were also observed (Fig. 1C and E). The papillary dermis exhibited large dark areas, representing blood vessels containing white central elements corresponding to erythrocytes, and bright perivascular elements of inflammatory infiltrate (Fig. 1E). Staining with hematoxylin-eosin disclosed features highly suggestive for the diagnosis of $\mathrm{AC}$ (Fig. 1F). The postoperative results were excellent, visible in the 7-month follow-up clinical photograph (Fig. 1G), without any indication of local recurrence. The patient was instructed to continue rigorous photoprotection, and further follow-up visits were scheduled.

Case 2. A 65-year-old Caucasian male, without any remarkable personal or family medical history, was referred in 2017 from the Department of Oral and Maxillofacial Surgery of 'Dr Carol Davila' Central Military Hospital in Bucharest to the Dermatology Department of 'Prof. N.C. Paulescu' National Institute of Diabetes, Nutrition and Metabolic Diseases, Bucharest, for the evaluation of two adjacent tumors growing on his lower lip. A clinical examination revealed an increased lower lip volume, and two central, asymptomatic, well-demarcated, round-to-oval tumors, with diameters of $20 \mathrm{~mm}$ and $5 \mathrm{~mm}$, 

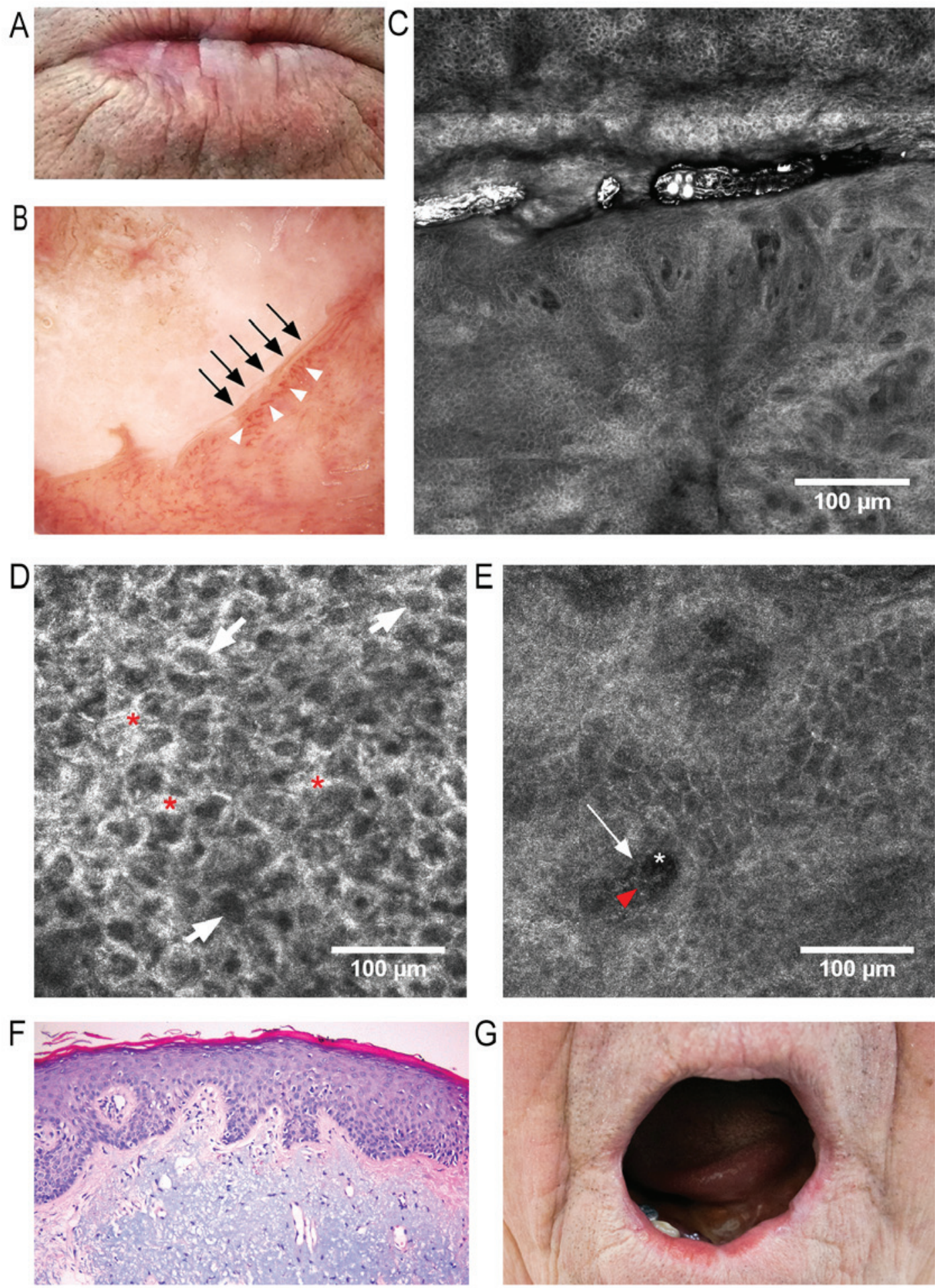

Figure 1.f (A) Clinical image: White keratotic areas on the lower lip surface with blurring of skin-vermillion contour. (B) Dermoscopic image: Milky-white plaque with well-defined borders (black arrows) surrounded by dilated and tortuous vessels (white arrowheads). (C) RCM block (dimensions: $2.5 \times 2.5 \mathrm{~mm}$ ) showing, in the upper third, a keratotic area with impaired keratinocyte architecture, enlarged intercellular spaces and atypical honeycomb appearance, and in the lower two-thirds, dilated blood vessels with tortuous aspect and bright perivascular inflammatory cells. (D) RCM image (500x500 $\mu \mathrm{m})$ at the stratum spinosum, showing atypical honeycombed pattern, enlarged intercellular spaces (red asterisks), and dark central elements representing nuclei surrounded by bright cytoplasm (white arrows). (E) RCM image (500x500 $\mu \mathrm{m}$ ) at the dermo-epidermal junction (DEJ)/papillary dermis, revealing dark areas representing papillary blood vessels (white asterisk) containing central elements corresponding to blood cells (red arrowhead) and bright perivascular structures equivalent to perivascular inflammatory cells (white arrow). (F) Histopathological image (hematoxylin and eosin staining; magnification, x200) of AC, showing hyperkeratosis and irregular acanthosis, mildly atypical keratinocytes within the lower third of epithelium, and marked solar elastosis and vascular ectasia within the dermis. (G) Clinical image captured 7 months after classic vermilionectomy, with minimal scarring and deformity, and with no signs of recurrence. RCM, reflectance confocal microscopy.

respectively. The larger lesion was brown-to-dark red in color, whereas both tumors presented keratotic surfaces and several ulcerations and crusts. Areas of ulceration and a significant amount of crusting on the remaining lower lip surface could 
be observed, with approximately two-thirds of the lip being affected, all on a background of visible vermillion atrophy (Fig. 2A). Dermoscopy of the tumors revealed marked vascular polymorphism, including telangiectatic, branching blood vessels, as well as hairpin, serpiginous, truncated and dotted vessels. Brown and white structureless areas were also visible. The surrounding lip surface presented slight-to-moderate vascular polymorphism, with dotted and short, anastomosing vessels (Fig. 2B). Dermoscopy of the perilesional area revealed ulcerations of the mucosa, surrounded by milky-white areas, telangiectatic and tortuous vessels, suggestive of AC (Fig. 2C). Upon RCM examination, an RCM block of the larger lower lip lesion (Fig. 2D) uncovered a disorganized keratinocyte architecture and loss of the normal honeycombed pattern with extensive keratinocyte atypia, markedly dilated blood vessels (Fig. 2E), and bright inflammatory cells (Fig. 2F). Aside from keratinocyte pleomorphisms and enlarged intercellular spaces, a particular 'swirl' was noticed, most likely due to incomplete keratinization (Fig. 2G). RCM images of the perilesional area revealed erosions surrounded by an appreciable amount of bright inflammatory cells (Fig. 2H). The tumors were completely excised with oncologically safe surgical margins and a histopathological examination (using hematoxylin-eosin staining) revealed, in the perilesional area, features highly suggestive for the diagnosis of AC (Fig. 2I), whereas an examination of the excised tumors confirmed the diagnosis of moderately differentiated invasive SCC (Fig. 2J). On the second day following vermilionectomy, the wound was healing well, with a minimal amount of crusting (Fig. 2K). At 5 weeks following surgery, there was minimal scarring and deformity of the lower lip (Fig. 2L). Due to the surgical technique employed, there was significant sparing of healthy tissue, which allowed for the avoidance of microstomia and its cosmetic and functional consequences in this patient.

\section{Discussion}

Due to the subtle nature of the initial clinical manifestations of $\mathrm{AC}$, confirming the diagnosis is of overwhelming importance. Furthermore, no correlations between the clinical aspect and histopathological changes have been established thus far (24), and there are no clear-cut clinical characteristics that separate AC from early SCC of the lips $(12,18)$.

Dermoscopic criteria for AC diagnosis include ill-demarcated lesion borders, white-coloured projections, 'island'-like structures and radially arranged vascular telangiectasia surrounding ulcerated areas (41). In the present case report, a milky-white plaque with well-defined borders surrounded by telangiectatic and tortuous vessels was identified in our patient (Case 1). Classically described dermoscopic features of SCC are glomerular or dotted vessels against a red, scaly background $(32,42)$. In our other patient (Case 2), dermoscopy revealed marked vascular polymorphism (telangiectatic, branching, hairpin, serpiginous, truncated and dotted blood vessels), and white, structureless areas.

RCM criteria for AC include disruption of the stratum corneum, single detached corneocytes and parakeratosis, an atypical honeycomb pattern with variation in cell size and morphology in the stratum granulosum and spinosum, dermal solar elastosis described as bright dense bundles with lace-like appearance, and dilated and tortuous dermal blood vessels with increased blood flow. It is also considered that keratinocyte atypia and an atypical honeycomb pattern serve as the most important features for AC diagnosis (19). The findings of the present case studies support the observation (19) that keratinocyte atypia and an atypical honeycomb pattern represent the most important diagnostic criteria of AC. True atypia as observed in AC displays cells and nuclei of different shapes and sizes, creating an atypical honeycomb pattern on RCM that must be distinguished from spongiosis.

Regarding RCM evaluation of lip SCC, Rishpon et al (32) reported certain distinct features, such as extensive atypia and disarrangement of the spinous and granular layer, round bright nucleated cells (corresponding to atypical and dyskeratotic keratinocytes observed on histopathological slides), and atypical nucleated cells in the superficial dermis (32). In our case of lip SCC, severe disarrangement of the spinous layer, extensive keratinocyte atypia, round, dilated and tortuous blood vessels accompanied by perivascular inflammatory infiltrates, dermal solar elastosis, and an abundant inflammatory infiltrate were observed.

In clinical practice, troubles associated with visual detection of carcinoma and dysplasia margins could be mediated by analysis of frozen sections from the lesion edge, but this is costly, time-consuming, conditional on the experience of the pathologist, and it is not widely available (43). Hence, non-invasive imaging technologies, such as RCM and dermoscopy, may allow an accurate identification of tumor margins in real time, adding substantial benefits for patients. RCM clearly has the potential to evaluate features of normal mucosa, dysplasia and lip SCC, and may provide a very appealing alternative for mucosal margins assessment due to its ability to resolve cellular morphology and tissue architecture in real time (44).

Numerous surgical and non-surgical strategies have been devised for the management of AC. Common treatment options of AC include cryosurgery, photodynamic therapy, $50 \%$ trichloroacetic acid chemical peel, $\mathrm{CO}_{2}$ laser ablation, electrodessication, and vermilionectomy. Topical treatments include applications of 5-fluorouracil, imiquimod, and 3\% Diclofenac in $2.5 \%$ hyaluronic acid (45-47). One of the classical approaches for the management of lip SCC is considered to be surgical excision $(3-5,48)$. In the present case report, we opted for surgical excision via vermilionectomy, given the fact that it provides a safe, quick, and economically efficient therapeutic option, particularly in the case of lower lip SCC. The two patients have been scheduled for regular follow-up visits.

In conclusion, due to the high rate of malignant transformation of AC into SCC of the lips, because of its great clinical variability, and since, for the majority of patients, it appears to be innocuous, the gravity of AC may, in a large number of cases, be underestimated, and all methods contributing to early diagnosis and dynamic monitoring of lesions must be employed. Considering how the majority of skin lesions are effortlessly assessed by clinical examination and dermoscopy, what role does RCM serve in patient care? Even though RCM might be considered time-consuming, in our experience image acquisition only required $\sim 10$ min per lesion, but markedly increased our diagnostic confidence, thereby limiting medical errors. 

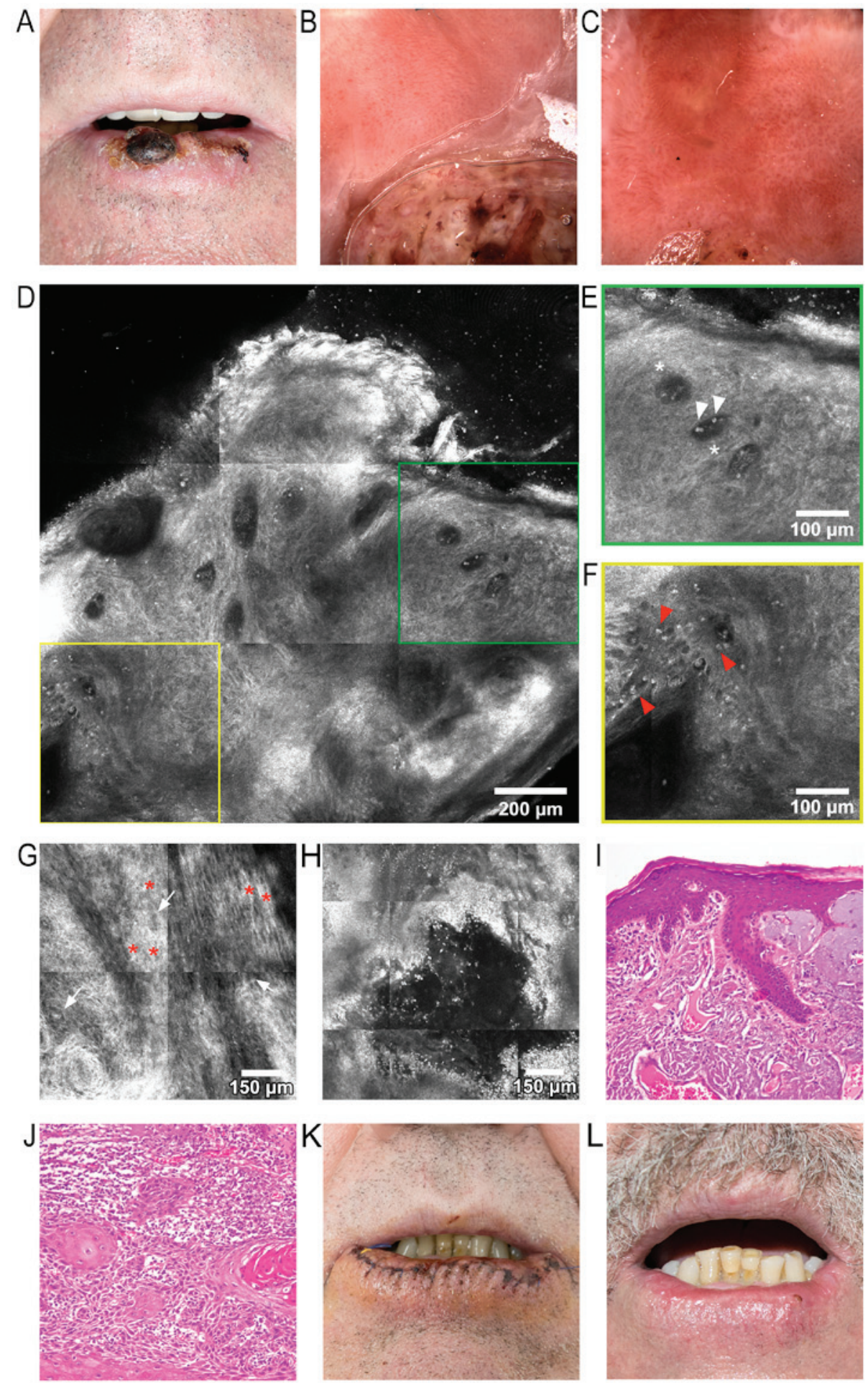

Figure 2. (A) Clinical image, showing the two tumors on the lower lip, with approximately two thirds of the lower lip exhibiting clinical signs of actinic cheilitis. (B) Dermoscopic image of the larger lower lip tumor: Part of the tumor is observed in the lower half, showing marked vascular polymorphism, including telangiectatic, branching blood vessels, as well as hairpin, serpiginous, truncated and dotted blood vessels. (C) Dermoscopic image of the perilesional area, revealing ulcerations of the mucosa surrounded by milky-white areas, and telangiectatic and tortuous vessels. (D) RCM block (dimensions: $1.5 \times 1.5 \mathrm{~mm}$ ), showing a disorganized keratinocyte architecture and loss of the normal honeycomb pattern, markedly dilated blood vessels, and scattered bright elements representing inflammatory cells. (E) RCM detail image $(500 \times 500 \mathrm{~mm}$ ) from (D), showing dark areas corresponding to dilated blood vessels (white asterisks) containing moderately refractile elements (white arrowheads). (F) RCM detailed image (500x500 mm) from (D), depicting white dots and plump refractile elements (red arrowheads) corresponding to inflammatory cells. (G) RCM block $(1 \mathrm{x} 1 \mathrm{~mm})$ at the stratum spinosum, demonstrating keratinocitary pleomorphisms of shape and size (white arrows); thickened intercellular spaces, equivalent to spongiosis, can be observed (red asterisks), and a particular 'swirl' effect in the lower-left corner of the image. (H) RCM block $(1 \mathrm{x} 1 \mathrm{~mm})$ of the perilesional area, revealing an ulceration and multiple bright elements corresponding to inflammatory cells. (I) Histopathological image (hematoxylin-eosin staining; magnification, x200) of actinic keilitis, showing epidermis thickened by hyperkeratosis, acanthosis and irregular elongation of rete ridges, several atypical keratinocytes present in the lower third of the epithelium, subjacent dermis with fibrosis, several ectatic blood vessels and marked solar elastosis. (J). Histopathological image (hematoxylin-eosin staining; magnification, x200) of moderately differentiated invasive squamous cell carcinoma: Trabeculi, and nests of moderately pleomorphic atypical squamoid cells with occasional parakeratotic pearl-like structures. (K) Clinical photograph captured 2 days after vermilionectomy. (L) Clinical image of the patient 5 weeks after surgery; there is minimal scarring and deformity of the lower lip visible at this time. RCM, reflectance confocal microscopy. 
Taking all our findings together, the present authors stress the importance of a correct diagnosis, proper treatment and longterm patient follow-up as indispensable for impeding the development of SCC of the lips, or for its early diagnosis.

\section{Acknowledgements}

Not applicable.

\section{Funding}

This study was partly supported by grant no. PN-III-P1-1.2PCCDI-2017-0341, financed by Executive Agency for Higher Education, Research, Development and Innovation.

\section{Availability of data and materials}

Data sharing is not applicable to this article, as no datasets were generated or analyzed during the current study.

\section{Authors' contributions}

All authors have equally contributed to writing and editing the manuscript.

\section{Ethics approval and consent to participate}

The two patients provided their informed consent for this study, which also included their consent to publish the results from their treatment.

\section{Consent for publication}

All authors have read and approved this manuscript.

\section{Competing of interests}

The authors declare they have no competing interests.

\section{References}

1. de Souza Lucena EE, Costa DCB, da Silveira EJD and Lima KC: Prevalence and factors associated to actinic cheilitis in beach workers. Oral Diseases 18: 575-579, 2012.

2. Strengthening the prevention of oral cancer: the WHO perspective. Community Dent Oral Epidemiol 33: 397-399, 2005.

3. Baker S: Current management of cancer of the lip. Oncology (Williston Park, NY) 4: 107-120; discussion 122-104, 1990.

4. Stucker FJ and Lian TS: Management of cancer of the lip. Operative Techniques in Otolaryngology Head and Neck Surgery 15: 226-233, 2004.

5. Zitsch RP, 3rd: Carcinoma of the lip. Otolaryngol Clin North Am 26: 265-277, 1993.

6. Galyon SW and Frodel JL: Lip and perioral defects. Otolaryngol Clin North Am. 34: 647 666, 2001.

7. Boda D, Neagu M, Constantin C, et al: HPV strain distribution in patients with genital warts in a female population sample. Oncol Let 12: 1779-1782, 2016.

8. Neagu M, Caruntu C, Constantin C, et al: Chemically induced skin carcinogenesis: Updates in experimental models (Review) Oncol Rep 35: 2516-2528, 2016.

9. Wood NH, Khammissa R, Meyerov R, Lemmer J and Feller L: Actinic cheilitis: a case report and a review of the literature. Eur J Dent 5: 101-106, 2011

10. Kwon NH, Kim SY and Kim GM: A case of metastatic squamous cell carcinoma arising from actinic cheilitis. Ann Dermatol 23 : 101-103, 2011.
11. Miranda AM, Soares LG, Ferrari TM, Silva DG, Falabella ME and Tinoco E: Prevalence of actinic cheilitis in a population of agricultural sugarcane workers. Acta Odontol Latinoam. 25: 201-207, 2012.

12. Miranda AM, Ferrari T, Leite T, Domingos T, Cunha K and Dias E: Value of videoroscopy in the detection of alterations of actinic cheilitis and the selection of biopsy areas. Med Oral Patol Oral Cir Bucal 20: e292-e297, 2015.

13. Kaugars GE, Pillion T, Svirsky JA, Page DG, Burns JC and Abbey LM: Actinic cheilitis: A review of 152 cases. Oral Surg Oral Med Oral Pathol Oral Radiol Endod 88: 181-186, 1999.

14. Vieira RAMAR, Minicucci EM, Marques MEA and Marques SA: Actinic cheilitis and squamous cell carcinoma of the lip: clinical, histopathological and immunogenetic aspects. An Bras Dermatol 87: 105 114, 2012.

15. Savage NW, McKay C and Faulkner C: Actinic cheilitis in dental practice. Australian dental journal 55 Suppl 1: 78-84, 2010.

16. Cavalcante ASR, Anbinder AL and Carvalho YR: Actinic Cheilitis: Clinical and Histological Features. J. Oral Maxillofac. Surg. 66: 498 503, 2008.

17. de Santana Sarmento DJ, da Costa Miguel MC, Queiroz LM, Godoy GP and da Silveira EJ: Actinic cheilitis: clinicopathologic profile and association with degree of dysplasia. Int J Dermatol 53: 466 472, 2014.

18. Markopoulos A, Albanidou Farmaki E and Kayavis I: Actinic cheilitis: clinical and pathologic characteristics in 65 cases. Oral Diseases 10: 212 216, 2004.

19. Ulrich M, Gonzalez S, Lange Asschenfeldt B, et al: Non invasive diagnosis and monitoring of actinic cheilitis with reflectance confocal microscopy. Journal of the European Academy of Dermatology and Venereology : JEADV 25: 276 284, 2011

20. Rivera $\mathrm{C}$ and Venegas $\mathrm{B}$ : Histological and molecular aspects of oral squamous cell carcinoma (Review). Oncol. Lett. 8: 7 11, 2014.

21. Miranda AMO, Ferrari TM and Calandro TLL: Actinic cheilitis: Clinical aspects and prevalence found in a rural population of the interior of Brazil. Saúde Pesqui 4: 67-72, 2011 (In Portuguese).

22. Lopes ML, Silva Junior FL, Lima KC, Oliveira PT and Silveira EJ: Clinicopathological profile and management of 161 cases of actinic cheilitis. An Bras Dermatol 90: 505-512, 2015.

23. Piñera Marques K, Lorenço SV, Silva LF, Sotto MN and Carneiro PC: Actinic lesions in fishermen's lower lip: clinical, cytopathological and histopathologic analysis. Clinics 65: 363-367, 2010.

24. Menta Simonsen Nico M, Rivitti EA and Lourenço SV: Actinic cheilitis: histologic study of the entire vermilion and comparison with previous biopsy. J Cutan Pathol 34: 309-314, 2007.

25. de Abreu MAMM, da Silva OMP, Pimentel DRN, et al: Actinic cheilitis adjacent to squamous carcinoma of the lips as an indicator of prognosis. Braz J Otorhinolaryngol 72: 767-771, 2006.

26. Glogau RG: The risk of progression to invasive disease. J Am Acad Dermatol 42: S23-S24, 2000.

27. Slaughter DP, Southwick HW and Smejkal W: 'Field cancerization' in oral stratified squamous epithelium. Clinical implications of multicentric origin. Cancer 6: 963-968, 1953.

28. Lupu M, Caruntu C, Ghita MA, et al: Gene Expression and Proteome Analysis as Sources of Biomarkers in Basal Cell Carcinoma Dis Markers 2016: 1-9, 2016.

29. Ion A,Popa IM, Papagheorghe LML, et al: Proteomic Approaches to Biomarker Discovery in Cutaneous T Cell Lymphoma. Dis Markers 2016: 1-8, 2016.

30. Voiculescu V, Calenic B, Ghita M, et al: From Normal Skin to Squamous Cell Carcinoma: A Quest for Novel Biomarkers. Dis Markers 2016: 1-14, 2016.

31. White WM, Rajadhyaksha M, González S, Fabian RL and Anderson RR: Non-invasive Imaging of Human Oral Mucosa in vivo by Confocal Reflectance Microscopy. Laryngoscope 109: 1709-1717, 1999.

32. Rishpon A, Kim N, Scope A, et al: Reflectance confocal microscopy criteria for squamous cell carcinomas and actinic keratoses. Archives of dermatology 145: 766-772, 2009.

33. Rajadhyaksha M, González S, Zavislan JM, Rox Anderson R and Webb RH: In Vivo Confocal Scanning Laser Microscopy of Human Skin II: Advances in Instrumentation and Comparison With Histology11The authors have declared conflict of interest. J Invest Dermatol 113: 293-303, 1999.

34. Ghita MA, Caruntu C, Rosca AE, et al: Reflectance confocal microscopy and dermoscopy for in vivo, non invasive skin imaging of superficial basal cell carcinoma. Oncol Lett 11: 3019-3024, 2016. 
35. Căruntu C1, Boda D, Guţ DE and Căruntu A: In vivo reflectance confocal microscopy of basal cell carcinoma with cystic degeneration. Rom J Morphol Embryol 55: 1437-1441, 2014

36. Căruntu C, Boda D, Căruntu A, Rotaru M, Baderca F and Zurac S: In vivo imaging techniques for psoriatic lesions. Rom J Morphol Embryol 55: 1191-1196, 2014.

37. Diaconeasa A, Boda D, Neagu M, Constantin C, Căruntu C, Vlădău L and Guţu D: The role of confocal microscopy in the dermato-oncology practice. J Med Life 4: 63-74, 2011.

38. Căruntu C and Boda D: Evaluation through in vivo reflectance confocal microscopy of the cutaneous neurogenic inflammatory reaction induced by capsaicin in human subjects. J Biomed Opt 17: 085003,2012.

39. Ridg way JM, Armstrong WB, Guo S, Mahmood U, Su J, Jackson RP, Shibuya T, Crumley RL, Gu M, Chen Z, et al: In vivo optical coherence tomography of the human oral cavity and oropharynx. Arch Otolaryngol Head Neck Surg 132: 1074-1081, 2006.

40. García-Hernández A, Roldán-Marín R, Iglesias-Garcia P and Malvehy J: In vivo noninvasive imaging of healthy lower lip mucosa: a correlation study between high definition optical coherence tomography, reflectance confocal microscopy, and histology. Dermatol Res Pract 2013: 205256, 2013.

41. Ito $T$, Natsuga $K$, Tanimura $S$, Aoyagi $S$ and Shimizu $H$ : Dermoscopic features of plasma cell cheilitis and actinic cheilitis Acta Derm Venereol. 94: 593 594, 2014.

42. Zalaudek I, Argenziano G, Leinweber B, et al: Dermoscopy of Bowen's disease. Br J Dermatol 150: 1112-1116, 2004

43. Nicoletti G, Brenta F, Malovini A, Musumarra G, Scevola S and Faga A: Study to determine whether intraoperative frozen section biopsy improves surgical treatment of non melanoma skin cancer. Mol Clin Oncol 1: 390 394, 2013.
44. Clark AL, Gillenwater AM, Collier TG, Alizadeh Naderi R, El Naggar AK and Richards Kortum RR: Confocal microscopy for real time detection of oral cavity neoplasia.Clin Cancer Res 9: 4714-4721, 2003.

45. Shah AY, Doherty SD and Rosen T: Actinic cheilitis: a treatment review. Int J Dermatol 49: 1225-1234, 2010

46. Ulrich C, Forschner T, Ulrich M, Stockfleth E, Sterry W and Termeer C: Management of actinic cheilitis using diclofenac 3\% gel: a report of six cases. Br J Dermatol 156: 43-46, 2007.

47. Matei C, Tampa M, Caruntu C, Ion RM, Georgescu SR, Dumitrascu GR, Constantin C and Neagu M: Protein microarray for complex apoptosis monitoring of dysplastic oral keratinocytes in experimental photodynamic therapy. Biol Res 47: 33, 2014.

48. Calabrese L, Ionna F, Tradati N, et al: Squamous cell carcinoma of the upper lip analysis of 123 cases. Int J Oncol 3: 667-669, 1993.

This work is licensed under a Creative Commons Attribution-NonCommercial-NoDerivatives 4.0 International (CC BY-NC-ND 4.0) License. 